This document is the Accepted Manuscript version of a Published Work that appeared in final form in Biomacromolecules, copyright $\odot$ American Chemical Society after peer review and technical editing by the publisher. To access the final edited and published work see https://10.1021/acs.biomac.6b01770.

Public access - Manuscript accepted

Published in final edited form as: Biomacromolecules 18 (1): 303-310, 2017

DOI : 10.1021/acs.biomac.6b01770

\title{
COMBINING ELECTROSPUN FIBER MATS AND BIOACTIVE COATINGS FOR USE IN VASCULAR GRAFT PROSTHESES
}

Houman Savoji ${ }^{1,2,5}$, Marion Maire², Pauline Lequoy ${ }^{2,4}$, Benoît Liberelle ${ }^{3}$, Gregory De Crescenzo $^{1,3}$, Abdellah Ajji1 ${ }^{1,3}$, Michael R. Wertheimer ${ }^{1,5}$, Sophie Lerouge ${ }^{2,4, *}$

École Polytechnique de Montréal, Montreal, QC, Canada ('Institute of Biomedical Engineering, ${ }^{3}$ Department of Chemical Engineering, ${ }^{5}$ Department of Engineering Physics)

${ }^{2}$ Laboratory of Endovascular Biomaterials (LBeV), Research Centre, Centre Hospitalier de l'Université de Montreal (CRCHUM), Montreal, QC, Canada

${ }^{4}$ Department of Mechanical Engineering, École de technologie supérieure, Montreal, QC, Canada

\section{Corresponding author:}

Sophie Lerouge, Department of Mechanical Engineering, École de technologie supérieure, 1100 Notre-Dame Ouest, Montreal, QC, H3C 1K3, Canada

E-mail: sophie.lerouge@etsmtl.ca 


\begin{abstract}
The patency of small-diameter ( $<6 \mathrm{~mm}$ ) synthetic vascular grafts (VGs) is still limited by the absence of a confluent, blood flow-resistant monolayer of endothelial cells (ECs) on the lumen, and of vascular smooth muscle cell (VSMC) growth into the media layer. In this research, electrospinning has been combined with bioactive coatings based on chondroitin sulfate (CS) to create scaffolds that possess optimal morphological and bioactive properties for subsequent cell seeding. We fabricated random and aligned electrospun poly(ethylene terephthalate), ePET, mats with small pores (3.2 \pm 0.5 or $3.9 \pm 0.3 \mu \mathrm{m}$,), then investigated the effects of topography and bioactive coatings on EC adhesion, growth and resistance to shear stress. Bioactive coatings were found to dominate the cell behavior, enabling creation of a near-confluent EC monolayer that resisted physiological shear-flow conditions. CS is particularly interesting since it prevents platelet adhesion, a key issue to avoid blood clot formation in case of an incomplete EC monolayer or partial cell detachment. Regarding the media layer, circumferentially oriented nano-fibers with larger pores $(6.3 \pm 0.5 \mu \mathrm{m})$ allowed growth, survival and inward penetration of VSMCs, especially when the CS was further coated with tethered, oriented epidermal growth factor (EGF). In summary, the techniques developed here can lead to adequate scaffolds for the luminal and media layers of small-diameter synthetic VGs.
\end{abstract}

Keywords: electrospinning; vascular graft; bioactive coatings; chondroitin sulfate; EGF immobilization; endothelialization; shear-induced flow; VSMC survival

\title{
1. Introduction
}

Primary requirements for functional synthetic small-diameter vascular grafts (VGs) or tissue engineered blood vessels for coronary or femoral artery bypass are favorable compliance and hemocompatibility [1]. To achieve long-term patency, a VG should thus possess a continuous and stable monolayer of endothelial cells (ECs) on its lumen, with significant resistance to physiological shear stress [2]. Yet, stable endothelialization of VGs remains a so-far unmet objective, in spite of great efforts during the past decade. In vitro seeding of autologous ECs or in situ capture of endothelial progenitor cells on the luminal layer of grafts have been tested [3], but 
due to weak EC adhesion the implanted grafts lacked adequate long-term patency. This is partly due to the inability of conventional VGs to reproduce the morphology and cell-matrix interactions of native tissues. In the extracellular matrix (ECM), topographical cell guidance is assured thanks to various combinations of pores, ridges and fibers at micro/nanoscale levels. Additionally, the ECM is known to orchestrate time- and spatially-controlled delivery of biochemical cues that trigger specific cell signaling pathways to finely tune tissue growth and homeostasis $[4,5]$.

Electrospinning has been used to design highly porous scaffolds that mimic topological cues of the ECM within native arteries [6-8]. Nano/microfiber scaffolds with different degrees of fiberalignment and -diameter have therefore been developed to optimize structural and mechanical properties of VGs in an effort to regulate cell adhesion, shape, spreading, and migration [9-11].

Our team has investigated how electrospun polyethylene terephthalate (ePET) mat properties such as fiber diameter, orientation and surface characteristics affect EC adhesion, spreading, infiltration and migration [11-14]. PET was selected for its good mechanical properties, stability, biocompatibility, and low cost. Furthermore, large diameter woven PET VGs have already been FDA approved and clinically used for many years [1, 12, 13]. The results of above-cited studies demonstrated that mats with small diameter fibers were appropriate to form an EC monolayer on the lumen of a VG [11], but a completely confluent monolayer could not be achieved under shear stress $[7,12]$.

A promising method to further enhance EC adhesion, growth and resistance to shear stress is to combine electrospun fibers with a bioactive coating [15]. We have recently shown that a primary amine-rich, low-pressure plasma-polymerized coating (hereafter “LP”) was able to significantly increase EC growth and retention $[12,16]$, but that retention was still not entirely complete. Moreover, LP was found to promote platelet adhesion and aggregation, that is, increased risk of thrombosis if the endothelial lining partially detaches under shear [17]. Our team also demonstrated $[17,18]$ that chondroitin sulfate (CS), a sulfated glycosaminoglycan that is abundant in the ECM of native blood vessels [19], once grafted on LP coating, also promotes EC adhesion and growth, but strongly reduces protein adsorption, platelet adhesion and aggregation [17]. Therefore, we propose here to combine electrospun mats with a bioactive coating that includes CS, in order to achieve a complete, stable endothelial lining. 
Electrospinning may also help to achieve a porous structure for constructing the media layer of a synthetic blood vessel, a layer which contains circumferentially-aligned fibres and the vascular smooth muscle cells (VSMC) responsible for contraction, dilation and blood pressure control [20, 21]. Therefore, the combination of an electrospun scaffold with a bioactive coating was also evaluated here as a mean to enhance VSMC growth and infiltration into porous mats. In addition to CS, epidermal growth factor (EGF), a 6-kDa mitogenic protein known to enhance VSMC growth, was also tethered on CS-grafted surfaces in an oriented manner, because this strategy is known to improve GF potency compared with random covalent binding [22-24].

We here report the cooperative effects of topological and biochemical features (the degree of alignment of electrospun nano-fibers, and the use of bioactive coatings) using three-dimensional (3D) ePET nanofiber scaffolds, upon cell growth of luminal (EC) and media (VSMC) layers for VGs. First, we combined LP+CS coatings with smaller pore-size random (hereafter "RL") and aligned (hereafter "AL") ePET mats for the luminal layer, then evaluated their ability to favor human umbilical vein EC (HUVEC) adhesion, growth and resistance to shear-induced flow. Next, we combined bioactive coatings (LP+CS+oriented tethered EGF) with aligned mats having somewhat larger pore-size for the media layer (hereafter “AM"), and investigated their bioactivity and ability to favor VSMC adhesion, growth and infiltration.

\section{Materials and Methods}

\subsection{Materials}

PET pellets were obtained from DuPont selar PT 7086 (intrinsic viscosity of 1), and ethylene ( $\left.\mathrm{C}_{2} \mathrm{H}_{4} ; 99.5 \%\right)$ and ammonia gas ( $\left.\mathrm{NH}_{3}, 99.99 \%\right)$ from Air Liquide Canada Ltd. (Montreal, Canada). Chondroitin sulfate (CS), bovine serum albumin (BSA, $66 \mathrm{kDa}$ ) were purchased from SigmaAldrich Canada Ltd; DuoSet ELISA kits were obtained from R\&D Systems, Minneapolis, MN. When not mentioned otherwise, chemical products were purchased from Sigma-Aldrich Canada Ltd. 


\subsection{Fabrication of Random and Aligned Bioactive Nano-fibrous Scaffolds}

\subsubsection{Electrospinning}

Optimized ePET mats for luminal and medial layers of VGs were prepared on a rotating mandrel, using a homemade electrospinning set-up. As previously described [12, 14], PET pellets were dissolved in 1:1 mixture of analytical grade dichloromethane $\left(\mathrm{CH}_{2} \mathrm{Cl}_{2}\right)$ /trifluoro-acetic acid (TFA), and gently stirred for $24 \mathrm{~h}$ at room temperature. Two milliliters $(\mathrm{mL})$ of polymer solution were electrospun with the aid of a syringe pump, in an enclosure at ambient humidity and temperature. Electrospinning parameters (Table 1) were adjusted to fine-tune the morphology of the mats for HUVEC and VSMC culture, i.e. random and aligned ePET mats with smaller pores for the luminal layer (RL and AL), and aligned mat with larger pore-size for the media layer (AM). The mandrel velocity was increased to obtain more aligned fibers for the luminal layer. On the other hand, a partially aligned, larger-pore mat for the media layer was obtained using higher PET concentration, a larger needle and relatively high drum speed. The fabricated electrospun scaffolds were dried in ambient air for 3 days to remove any residual solvent.

Table 1 Process conditions used to produce the electrospun random and aligned ePET mats

\begin{tabular}{|c|c|c|c|c|c|c|c|c|}
\hline \multirow[b]{2}{*}{ Mat type } & \multirow{2}{*}{$\begin{array}{c}\text { PET solution } \\
\text { concentration } \\
(\% \mathrm{w} / \mathrm{v})\end{array}$} & \multicolumn{4}{|c|}{ Process parameters } & \multirow{2}{*}{$\begin{array}{c}\text { Collector } \\
\text { Mandrel } \\
\text { velocity } \\
\text { (RPM) }\end{array}$} & \multicolumn{2}{|c|}{$\begin{array}{l}\text { Ambient } \\
\text { conditions }\end{array}$} \\
\hline & & $\begin{array}{l}\text { Needle } \\
\operatorname{size}(G)\end{array}$ & $\begin{array}{l}\text { Collector } \\
\text { distance } \\
\text { (cm) }\end{array}$ & $\begin{array}{c}\text { Voltage } \\
(\mathrm{kV})\end{array}$ & $\begin{array}{c}\text { Flow } \\
\text { rate } \\
(\mathrm{mL} / \mathrm{h})\end{array}$ & & $\begin{array}{l}\text { RH } \\
(\%)\end{array}$ & $\operatorname{Temp}\left({ }^{\circ} \mathrm{C}\right)$ \\
\hline RL & 9 & 22 & 15 & $15-17$ & 0.5 & 175 & $8-25$ & $20-25$ \\
\hline AL & 9 & 22 & 15 & 20 & 0.5 & 2000 & $8-25$ & $20-25$ \\
\hline $\mathbf{A M}$ & 12.5 & 18 & 13 & 30 & 10 & 1000 & $8-25$ & $20-25$ \\
\hline
\end{tabular}


RL: random mat for luminal layer; AL: aligned mat for luminal layer; AM: aligned mat for media layer

\subsubsection{Plasma-Polymerization}

Primary-amine rich plasma polymer (LP) coatings were deposited on the surface of the nano-fiber mats in a low-pressure ("L”) capacitively-coupled radio-frequency (r.f., $13.56 \mathrm{MHz}$ ) glow discharge plasma reactor, using ethylene $\left(\mathrm{C}_{2} \mathrm{H}_{4}\right) /$ ammonia $\left(\mathrm{NH}_{3}\right)$ gas mixture $[25,26]$. The coatings were deposited under partial vacuum ( $p=80 \mathrm{~Pa}$ ) using constant flow rates of $\mathrm{C}_{2} \mathrm{H}_{4}$ "monomer" and ammonia, $\mathrm{NH}_{3}, \mathrm{FC}_{2} \mathrm{H}_{4}$ and $F_{\mathrm{NH}_{3}}$, with a mixture ratio $R=F_{\mathrm{NH}_{3}} / F_{\mathrm{C}_{2} \mathrm{H}_{4}}=0.75$. The deposition was performed for 8 min duration under mild plasma conditions (power, $P=10 \mathrm{~W}$, d.c. bias voltage, $V_{\mathrm{B}}=-40 \mathrm{~V}$ ). These conditions were previously shown to provide high nitrogen and amine concentrations, [N] and [ $\mathrm{NH}_{2}$ ], and good stability (insolubility) in aqueous solutions [26].

\subsubsection{CS Grafting on the LP-coated Mats}

Circular samples $\left(1 \mathrm{~cm}^{2}\right)$ of the amine-rich LP-coated ePET mats were punched to fit into 48-well plates. CS was dissolved in Milli-Q water at $0.1 \mathrm{~g} . \mathrm{mL}^{-1}$, and the resulting solution was filtered (200- $\mu \mathrm{m}$ PTFE filter) to remove aggregates. CS was covalently grafted on the mats by way of carbodiimide chemistry (NHS/EDC-based chemistry) [22]: The mats were incubated in $200 \mu \mathrm{L}$ of solution containing 0.01 g.mL $\mathrm{mL}^{-1} \mathrm{CS}, 40 \% \mathrm{v} / \mathrm{v}$ EtOH, $50 \mathrm{mM}$ MES, $22.8 \mathrm{mM}$ EDC, $4.6 \mathrm{mM}$ NHS for $1 \mathrm{~h}$ at room temperature, then finally rinsed once with PBS (10 mM, pH 7.4) and twice with Milli-Q water for $2 \mathrm{~min}$ in an ultrasonic bath.

\subsubsection{EGF Oriented Immobilization}

Oriented immobilization of EGF on CS-grafted surfaces was performed [22] using the Ecoil and Kcoil peptides that hetero-dimerize with high specificity and affinity [23, 27]. Cysteine-tagged Kcoil peptides were synthesized by the peptide facility at the University of Colorado (Denver, CO, USA). Ecoil-tagged EGF (E-EGF) was produced in human embryonic kidney (HEK) 293 cells and purified [28]. Kcoil was grafted on CS-grafted mats using EMCH linker that reacts with the carboxyl groups of CS and the thiol group of the cysteine-terminated Kcoil [22]. Mats covered with covalently bound Kcoil were incubated overnight in a 10\% solution of fetal bovine serum (FBS) in PBS to remove any unbound peptide. After rinsing with PBS, surfaces were incubated for $1 \mathrm{~h}$ with $200 \mu \mathrm{L}$ of E-EGF solution at $22 \mathrm{nM}$ concentration in $10 \mathrm{mM}$ PBS containing $1 \% \mathrm{BSA}$. 
This concentration was chosen based on our previous experiments on PET film surfaces [22]. After capture of the Ecoil-tagged protein, mats were rinsed with PBS.

\subsection{Characterization of Bioactive Scaffolds}

\subsubsection{Mat Morphology}

Electrospun nano-fiber structure and morphology were evaluated by scanning electron microscopy (SEM) using a JSM-7600 Field Emission instrument (JEOL Ltd., Tokyo, Japan) at $0.5 \mathrm{kV}$. Mat surfaces were first sputter-coated with gold for $45 \mathrm{~s}$; images were analyzed using ImageJ (NIH, USA) software, and diameters were calculated based on at least 100 fibers (at least two experiments with triplicate samples). The fiber alignment was characterized using the directional plugin of ImageJ to obtain Fast Fourier Transformation (FFT) of SEM images [14]. The porosity of nanofiber mats was determined using a liquid (ethanol) intrusion method [29]. In addition, the average pore size was evaluated by SEM with the ImageJ software, assuming elliptical pore shapes and taking an average of major and minor axes of the best-fitting ellipses.

\subsubsection{Chemical Composition}

X-ray photoelectron spectroscopy (XPS) analyses of bare and modified mats were performed in a VG ESCALAB 3MkII instrument, using non-monochromatic Mg K $\alpha$ radiation. To assess possible chemical composition gradients across the thickness of the mat, a two-layer scaffold was created by a two-step electrospinning approach. The first ply (ca. $50 \mu \mathrm{m}$, half of the final thickness) was fabricated and dried overnight; the second ply (approx. $50 \mu \mathrm{m}$ ) was then electrospun on top of the first. A LP+CS coating was created on top of the two-layer mat and XPS survey spectra were taken at different depths of this composite sample, namely, top ([a]: $0 \mu \mathrm{m}$ ), mid-plane ([b]: $50 \mu \mathrm{m}$ ), and bottom ([c]: $100 \mu \mathrm{m})$.

\subsubsection{Protein Adsorption Measurements}

Since CS has low-fouling properties [17], CS grafting throughout the scaffold thickness was examined by protein adsorption using Texas Red fluorescent-labeled bovine serum albumin (BSA), using the two-layer mat described above. Briefly, the two layers were gently separated and 
immersed in labeled BSA (0.2 mg/mL in PBS) for $2 \mathrm{~h}$ under static ambient conditions in the dark. Fluorescence intensity was measured after thorough washing with PBS to remove unbound protein. Images were analyzed using ImageJ software and the background auto-fluorescence was subtracted for each sample.

\subsubsection{E-EGF Quantification by ELISA}

E-EGF immobilized on the mats was quantified by direct enzyme-linked immunosorbent assay (ELISA) according to the manufacturer's instructions. The optical density (O.D.) was measured at $630 \mathrm{~nm}$. The slopes corresponding to the initial O.D. variation in the wells over time were calculated, and the value obtained for a surface with no growth factor was subtracted from all signals.

\subsection{Biological Testing}

\subsubsection{HUVEC Adhesion and Growth}

HUVECs (Cell Applications Inc.) were cultured in 1\% gelatin-coated flasks, in complete medium EGM-2 bullet Kit (Lonza, CC-3162) and used at passage 3 - 5. Random and aligned $1 \mathrm{~cm}$-diameter disk samples from bare, LP-coated, and CS-grafted mats were placed in 48-well polystyrene culture plates (hereafter "PCP”) and sterilized in 70\% ethanol for $5 \mathrm{~min}$, rinsed with sterile water, then dried overnight under a laminar hood. Cloning glass cylinders $(0.8 \mathrm{~cm}$ inner diameter, $1 \mathrm{~cm}$ height) were placed on the disk samples to prevent their movement after cell seeding and to prevent cell adhesion elsewhere. $200 \mu \mathrm{L}$ of HUVEC suspension (20,000 cells) was added to each well; as positive controls, cells were also cultured directly on PCP coated with 1\% gelatin. After a 4 h period of adhesion, cloning cylinders and medium were removed, and cells were left to grow for 1, 7, 14 and 21 days in $500 \mu \mathrm{L}$ of complete medium supplemented with 1\% penicillin-streptomycin (PenStrep; Invitrogen). HUVEC adhesion and growth on the various surfaces was evaluated using AlamarBlue (10 \%v/v) reduction assay (Cedarlane, 30025-1(BT), four samples each). The experiment was carried out in triplicate to ensure reproducibility. To assess cell viability and distribution in the 3D mats, Live-Dead assays were performed by incubating in calcein-AM (2 $\mu \mathrm{M})$ and ethidium homodimer-1 (5.5 $\mu \mathrm{M}$, Invitrogen) for $45 \mathrm{~min}$ at $37^{\circ} \mathrm{C}$ in the dark, before 
rinsing with PBS and imaging under a Laser Confocal Scanning Microscope (Olympus multiphoton FV-1000 MER, Olympus, Canada).

Immunostaining was performed to visualize actin microfilaments, focal adhesions and cell-cell contact points according to well-established procedures [16, 30]. Briefly, cells were fixed and permeabilized with $3.7 \%$ paraformaldehyde and $0.1 \%$ TRITON X-100 solution in PBS, respectively, then incubated with primary antibodies, namely anti-mouse vinculin (Millipore, MAB3574, diluted 1:100) and anti-mouse VE-cadherin (Millipore, diluted 1:100). Following this, the scaffolds were incubated with secondary antibody, goat anti-mouse Alexafluor 546 (Invitrogen, A-11018, diluted 1:200), then with the corresponding Alexa (Molecular Probes) Phalloidin Alexa-488 anti-mouse (Invitrogen, A-11029, diluted 1:40). Finally, cell nuclei were stained with Draq5 (diluted 1:1000). Samples were examined using a Leica TCS SP2 confocal laser-scanning microscope (Leica Microsystems, Wetzlar, Germany).

\subsubsection{Cell Alignment and Morphology}

Cell alignment was calculated from immunostained images using ImageJ software, based on the angle difference between the long axis of each nucleus (presumed elliptical) and the average fiber direction (100 nuclei in total), following which alignment angles were categorized in $10^{\circ}$ increments. Cells with angles $<30^{\circ}$ were considered aligned [31, 32]. In addition, the cell elongation was evaluated using ImageJ software by its shape index (circularity = $4^{*} \pi^{*}$ area/perimeter ${ }^{2}$ ) for each cell on random and aligned scaffolds, shape index 1 corresponding to a perfect circle [33]. SEM was also used to visualize cell morphology on controls and modified scaffolds.

\subsubsection{HUVEC Resistance to Laminar Shear Stress}

To study HUVEC resistance to shear, cells ( $250 \mu \mathrm{L}$ in complete medium, $10^{6}$ cells/mat) were seeded and left to adhere on mats $(10 \mathrm{~mm} \times 22.5 \mathrm{~mm})$ for $48 \mathrm{~h}$ so as to obtain a confluent monolayer. They were then subjected to laminar flow-induced shear stress of ca. 15 dynes $\mathrm{cm}^{-2}$ (similar to an actual physiological value [34]) for $1 \mathrm{~h}$ in a parallel-flow chamber (Glycotech, C 31-001, Gaithersburg MD) [12]. The influence of fiber alignment (random vs. aligned mats) and surface composition (bare, LP and LP+CS-coated mats) on cell retention was studied by AlamarBlue and SEM. 


\subsubsection{VSMC Adhesion, Growth and Survival}

VSMCs from rat embryonic thoracic aorta (a7r5 cell line, ATCC, VA, USA), cultured in Dulbecco’s Modified Eagle’s Medium/ Nutrient Mixture F-12 Ham’s Media (DMEM/F12; Invitrogen) supplemented with 10\% fetal bovine serum (FBS; Medicorp, Quebec, Canada), were used between passages 4 to 15. For cell growth and survival experiments, 20,000 and 30,000 cells/mat, respectively, were seeded in complete media on pristine and modified aligned mats (LP, $\mathrm{LP}+\mathrm{CS}, \mathrm{LP}+\mathrm{CS}+\mathrm{EGF}$ ), as was done in the case of HUVECs. Cell growth was assessed in complete media and cell survival in serum-free medium (DMEM/F12; Invitrogen), both using AlamarBlue. The media was changed every other day. For conditions designated as "soluble EGF", 10 ng.mL" ${ }^{1}$ of EGF was supplemented in the culture medium.

\subsubsection{Histology}

In order to evaluate cellular infiltration into the electrospun mats after 21 days of incubation, samples were rinsed with PBS and fixed with $10 \%$ neutral buffer formalin for $24 \mathrm{~h}$ at room temperature. After embedding in paraffin, $6 \mu \mathrm{m}$ thick microtomed sections were stained using Hematoxylin-Eosin (H\&E) and imaged.

\subsection{Statistical Analysis}

Each experiment was carried out in triplicate to examine reproducibility. All data are expressed as mean values \pm SD. Statistical analyses were carried out using ANOVA with Tukey's post hoc analysis, or by independent t-test when comparing two groups only. Values of $\mathrm{p}<0.05$ were considered significant for all tests.

\section{Results and discussion}

\subsection{Morphology of Random and Aligned Scaffolds for Lumen}

In blood vessels, ECs are well known to be aligned and elongated in the flow direction, which results in maximized cell retention under blood flow-induced shear stress [34]. To stimulate EC alignment by topographical cues, aligned scaffolds with small pore-size (AL) were compared with their random counterparts (RL) in regard to formation of an EC monolayer. SEM images (Figure 
1A and B) confirm uniform, smooth fiber morphology with interconnected open structure, and average diameters of ca. $550 \pm 90 \mathrm{~nm}$ and $500 \pm 140 \mathrm{~nm}$ for random and aligned nanofibers, respectively $(n=200)$. The overall porosity was similar $(87 \%)$ in both cases, mean respective pore diameters being ca. $3.2 \pm 0.5$ and $3.9 \pm 0.3 \mu \mathrm{m}$. The 2D FFT frequency images (Figure 1A and B, inset) show that only the aligned (AL) scaffolds exhibited an oriented structure. The fiber alignment was also quantified by the 2D FFT plot (see supplemental data Figure E1) which shows greatest intensity in the direction of fiber alignment on the micrograph, while sharp peaks at $90^{\circ}$ and $270^{\circ}$ confirm that the majority of fibers were oriented in a specific direction (perpendicular to the mandrel axis during electrospinning).

\subsection{Morphology of Aligned Scaffolds for Medial Layer}

Aligned scaffolds (AM) with greater pore size were fabricated in order to mimic the media layer (Figure 1C), respective average fiber diameter, pore diameter and porosity being $890 \pm 350 \mathrm{~nm}$, $6.3 \pm 0.5 \mu \mathrm{m}$ and $78 \%$. Greater pore sizes were desirable in this case to allow SMC infiltration into the bulk of the scaffold. The 2D FFT frequency image (Figure 1C, inset) and quantified plot (supplemental data Figure E1) also confirm the fiber alignment.
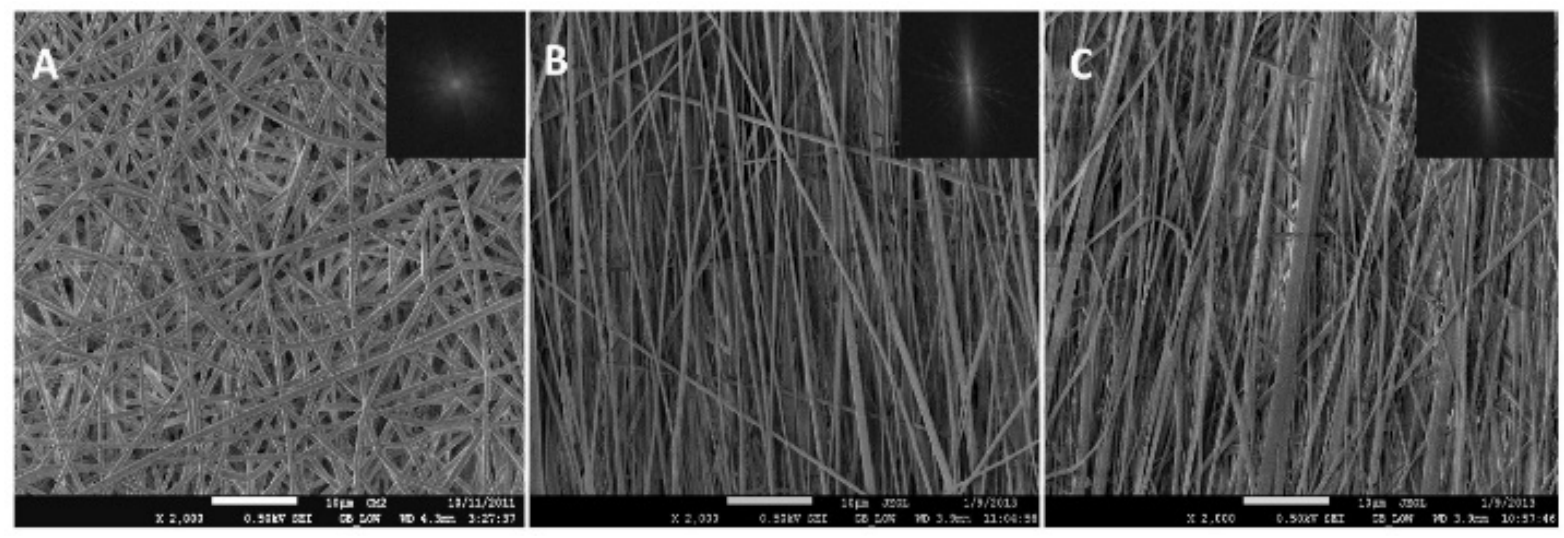

Figure 1 SEM and 2D FFT images (insets) of (A) random (RL), (B) aligned (AL) mat for the luminal layer; and (C) aligned (AM) mat for the media layer (scale bar: $10 \mu \mathrm{m}$ ). 


\subsection{Surface Chemistry and Depth-Dependence}

XPS-based elemental composition of an AM mat after LP deposition and CS grafting is presented in Table 2. From our earlier work it is known that LP is stable and that it comprises high values of $[\mathrm{N}]$ and $\left[\mathrm{NH}_{2}\right][12,35]$, reconfirmed here $([\mathrm{N}]=11.2 \%)$. The presence of sulfur $(\mathrm{S})$ also confirmed successful grafting of CS on the LP-coated mats. Furthermore, the ability to coat fibers with LP and LP+CS in the sample bulk was examined using the two-layer mat sample, on top [a]: $0 \mu \mathrm{m}$; in the middle, [b]: $50 \mu \mathrm{m}$; and at the bottom, [c]: $100 \mu \mathrm{m}(\mathrm{n}=3$, Table 2). Although [N] decreased with increasing depth, it was clearly measurable in cases [b] and [c], many tens of micrometers below the surface, confirming that active plasma species penetrated through the porous structure. After CS grafting, $[\mathrm{N}]$ decrease and [S] increase at each level of the mats are a first confirmation that CS was grafted across the mat thickness. 
Table 2 XPS-based chemical compositions of bare and coated aligned mats for the media layer (AM) and their depth-dependence $(n=3)$

\begin{tabular}{|c|c|c|c|}
\hline \multicolumn{4}{|c|}{ 2-layer AM bare mat } \\
\hline Depth & [a]: $0 \mu \mathrm{m}$ & [b]: $50 \mu \mathrm{m}$ & [c]: $100 \mu \mathrm{m}$ \\
\hline Elements & Avg. (\%) $\pm S D$ & Avg. (\%) $\pm S D$ & Avg. (\%) $\pm S D$ \\
\hline C & 70.5 & 70.5 & 70.5 \\
\hline $\mathbf{N}$ & 0 & 0 & 0 \\
\hline $\mathbf{O}$ & 29.5 & 29.5 & 29.5 \\
\hline $\mathbf{S}$ & 0 & 0 & 0 \\
\hline \multicolumn{4}{|c|}{ 2-layer AM+LP-coated mat } \\
\hline C & $84.3 \pm 1.4$ & $74.4 \pm 1$ & $74.5 \pm 0.8$ \\
\hline $\mathbf{N}$ & $11.2 \pm 0.9$ & $7.2 \pm 0.1$ & $5.1 \pm 0.8$ \\
\hline $\mathbf{O}$ & $4.6 \pm 2.4$ & $18.4 \pm 1.1$ & $20.4 \pm 0.0$ \\
\hline $\mathrm{S}$ & 0 & 0 & 0 \\
\hline \multicolumn{4}{|c|}{ 2-layer AM+LP+CS grafted mat } \\
\hline C & $69.3 \pm 1.4$ & $69.8 \pm 0.5$ & $71.3 \pm 1.4$ \\
\hline $\mathbf{N}$ & $8.7 \pm 1.2$ & $5.8 \pm 0.1$ & $4.4 \pm 0.3$ \\
\hline $\mathbf{O}$ & $21.3 \pm 0.2$ & $23.6 \pm 0.3$ & $23.6 \pm 1.2$ \\
\hline $\mathbf{S}$ & $0.7 \pm 0.1$ & $0.8 \pm 0.0$ & $0.8 \pm 0.1$ \\
\hline
\end{tabular}

\subsection{Protein Adsorption}

CS grafting throughout the thickness of the AM mats was confirmed by BSA adsorption on the same two-layer mats. Protein adsorption can serve as an indirect indicator for CS grafting thanks to the earlier-demonstrated low-fouling properties of CS [17]. As shown in Figure 2, LP coating 
increased albumin adsorption compared with bare ePET mats, as previously demonstrated on film substrates [17], while CS grafting significantly decreased it. As expected, these effects are less pronounced at the bottom (case [c]), which confirms lower $\left[\mathrm{NH}_{2}\right]$ after plasma (Table 2) and consequently less grafted CS.

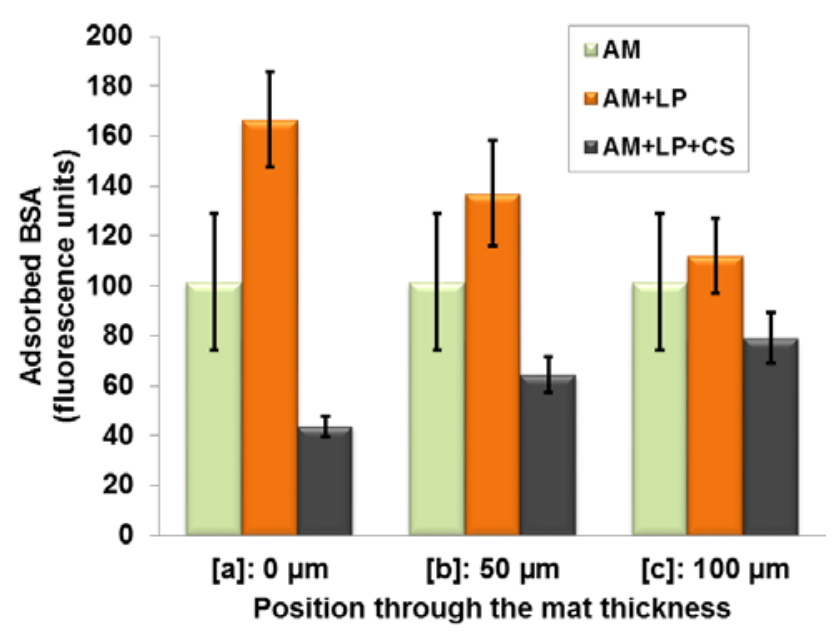

Figure 2 Fluorescence intensity of adsorbed Texas Red-BSA $(0.2 \mathrm{mg} / \mathrm{mL})$ on bare AM, $\mathrm{AM}+\mathrm{LP}-$, and AM+LP+CS-coated mats as a function of depth $(\mathrm{n}=10)$.

\subsection{Effect of Fiber Orientation and Bioactive Coatings on HUVECs}

Figure 3 presents adhesion and growth results for HUVECs on the various surfaces, assessed by their metabolic activity. Adhesion and growth on bare RL and AL scaffolds were found to be similar at each time point $(p>0.05)$. Interestingly, metabolic activity was found to drop sharply at day 7, but then it increased again, a type of behavior reported before by our team [12] and another group [36]. LP coating significantly increased cell adhesion and growth on both scaffolds ( $\mathrm{p}<$ 0.05 ), in agreement with previous studies on various $2 \mathrm{D}$ and 3D substrates [12, 16, 17]. CS-grafted scaffolds showed similar initial adhesion as LP ( $p>0.05)$, but more rapid growth thereafter, as shown by significantly higher values at day 7, 14 and 21 (Figure 3, Figure 4). 


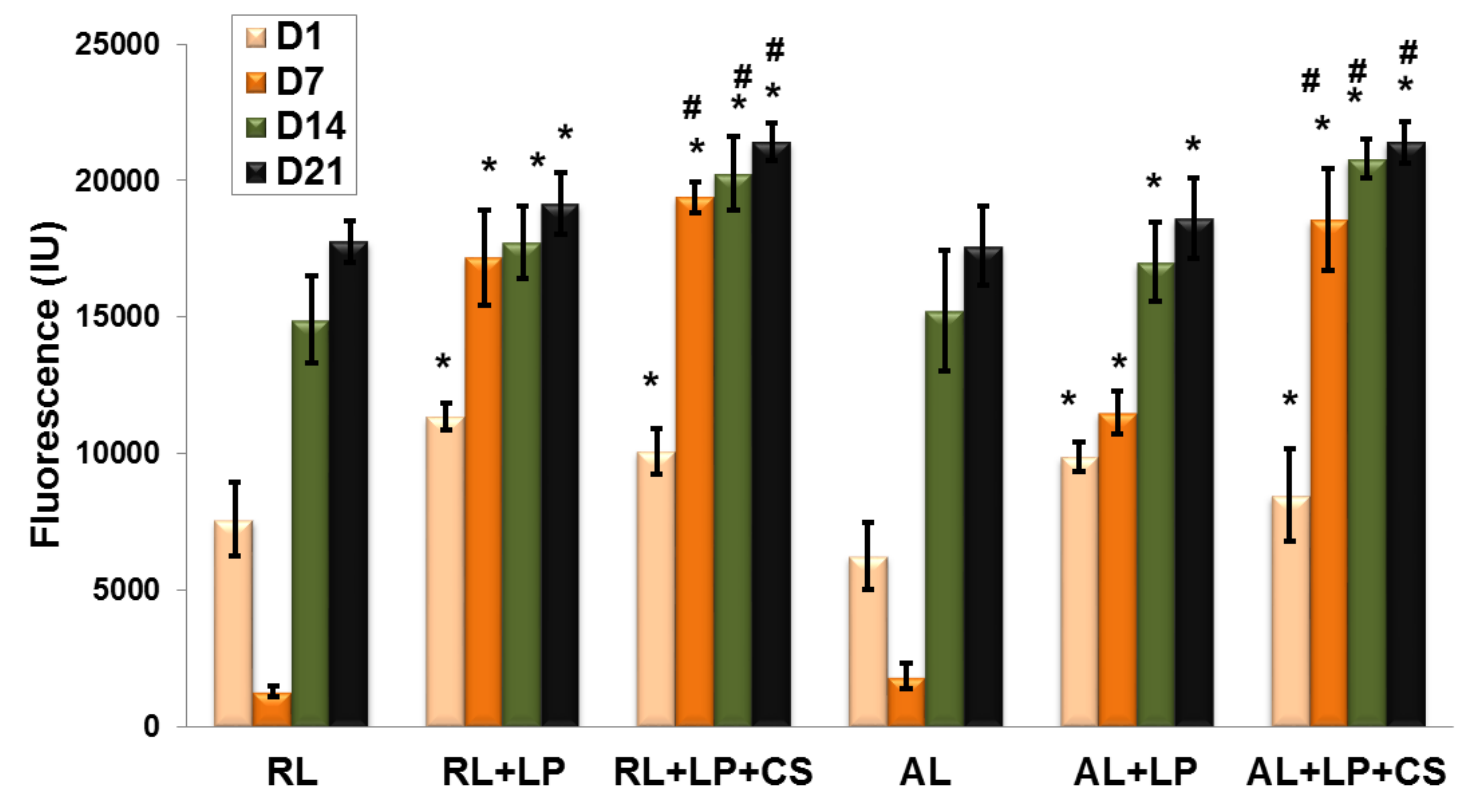

Figure 3 Adhesion (day 1) and growth (day 7, 14 and 21) of HUVECs on bare and coated random $(R L)$ and aligned $(A L)$ mats. Mean $\pm S D(n \geq 6)$.

( ${ }^{*} \mathrm{p}<0.05$ with bare surfaces; ${ }^{*} \mathrm{p}<0.05$ with LP-coated surfaces at the same time point).

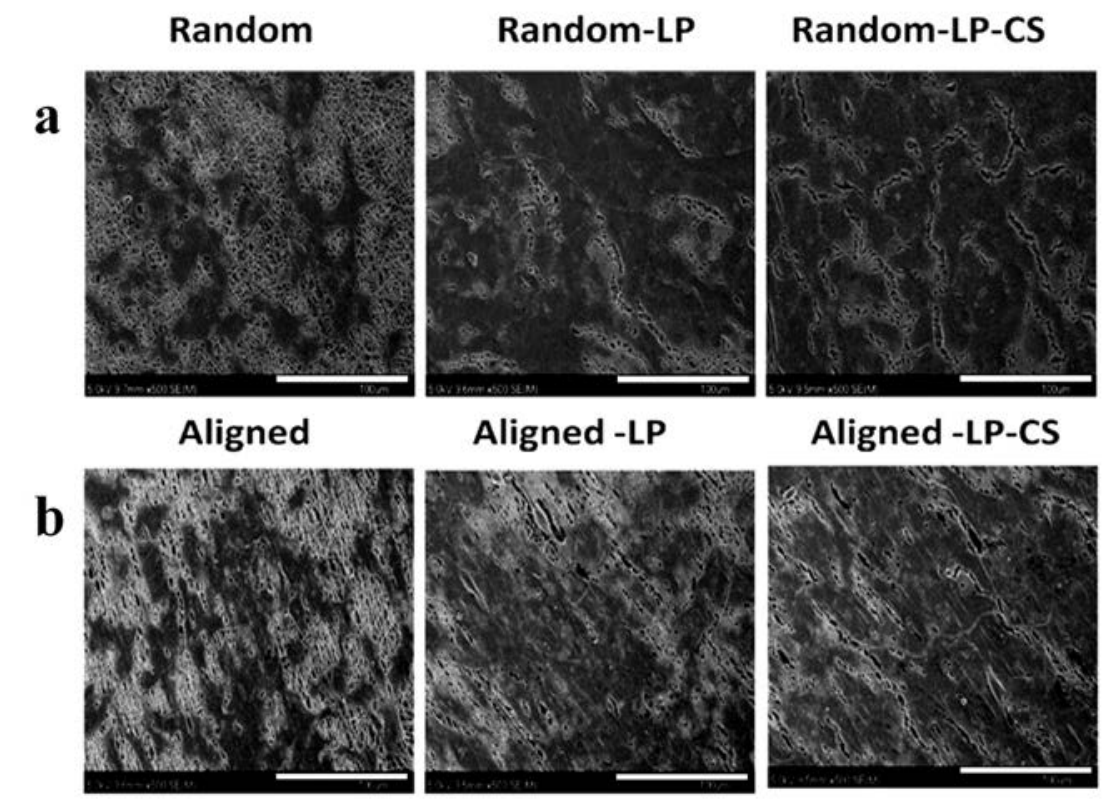

Figure 4 SEM images of HUVECs after 21 days of cell culture on a) bare and coated random (RL); b) bare and coated aligned (AL) ePET mats (scale bar: 100 $\mu \mathrm{m}$ ). 
The z-stack confocal images in Figure 5a confirm a monolayer of endothelial cells on both RL and AL scaffolds, with no sign of cell penetration into the bulk. Moreover, at locations where confluency occurred, VE-cadherin protein expression is noted at cell-to-cell contact regions, indicating that neighboring cells interacted in a coherent manner (Figure 5d). This would confirm that these nano-fibrous structures are indeed well adapted for forming confluent monolayers of endothelial cells.

Vinculin immunostaining showed no significant difference between the various mat morphologies. Even on the latter we observed only limited focal adhesion complexes, mostly at the polarized cell edges (Figure 5e). This agrees with other studies [37, 38], demonstrating weak focal points between cells and the electrospun nano- fibers.
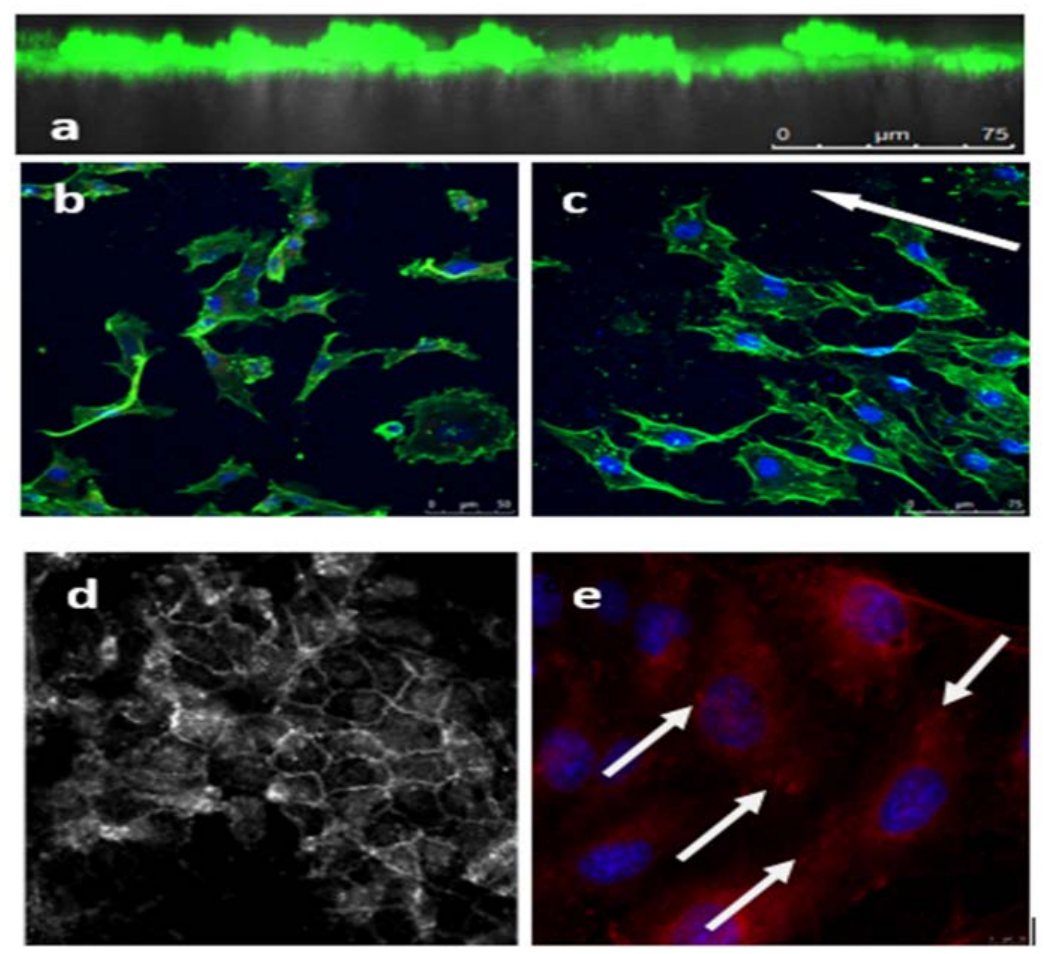

Figure 5 a) z-stack confocal micrograph of HUVECs grown (D21) on an AL mat (live cells in green); b, c) confocal micrographs of HUVEC adhered (at D1) on LP-coated (b) random (RL) and (c) aligned (AL) mats (cytoskeletal F-actin in green, nucleus in blue, electrospun fiber direction indicated by arrow); d) typical cell-cell interactions (VE-cadherin, in white); e) cell focal adhesion points (vinculin, in red) on LP-coated random (RL) mat at D7 (scale bar: $75 \mu \mathrm{m}$ ). 
Finally, cell retention after shear stress was tested on bare and coated mats (Figure 6). The percentage of cells remaining after shear was very low on bare mats (25-30\%), but much greater for LP and LP+CS coated ones, both random and aligned ( $\mathrm{LP}+\mathrm{CS}>\mathrm{LP}>$ bare; $\mathrm{p}<0.05)$. The performance was slightly (but not significantly) better on the aligned scaffolds, ones coated with LP+CS showing up to $82 \%$ cell retention.

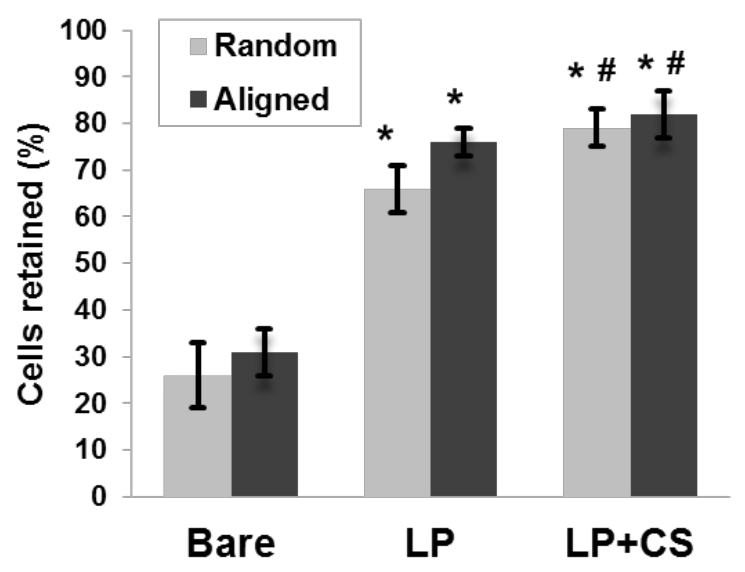

Figure 6 HUVEC retention after laminar shear stress (15 dynes.cm ${ }^{-2}$,1h), evaluated by AlamarBlue assay $(n=4)$. *, \# $\mathrm{p}<0.05$ with reference to bare and LP-coated surfaces under shear, respectively.

Some cell alignment was observed on the AL mats, as shown on Figure 5c by parallel-oriented Factin microfilaments, in contrast with the irregular cell shape observed in Figure $5 \mathrm{~b}$ for the random (RL) mats. This was confirmed by quantitative analysis of the nuclear alignment: $80 \%$ of the cells aligned with the AL fiber direction (angles $<30^{\circ}$, see supplemental data, Figure E2 and Table E1), contrary to random cell orientation for the RL scaffolds. Furthermore, there were significant differences in cell elongation between the RL and AL mats (circularity $67 \pm 12 \%$ versus $33 \pm 20 \%$, respectively, $\mathrm{p}<0.05)$. 


\subsection{VSMCs: Bioactivity of Coatings on Scaffolds for the Media Layer}

\subsubsection{Kcoil Grafting and EGF Capture - Quantification by ELISA}

For the media layer, EGF was additionally immobilized on a LP-CS coating. ELISA confirmed successful tethering of the EGF using the Kcoil-Ecoil system, the optical density on CS+Kcoil+EEGF being much greater than on other surfaces (9, 12 and 18-fold higher compared with simple CS, simple Kcoil and CS incubated with Ecoil EGF in absence of Kcoil, respectively, $\mathrm{p}<0.05$ ) (Figure E3, Supplemental data). This confirmed the specificity of the E/K coiled-coil interaction, as well as its suitability to capture EGF on CS.

\subsubsection{VSMC Adhesion and Growth}

Metabolic activity of VSMCs after 1 and 7 days on bare and coated mats is illustrated in Figure 7. At day 7, the number of cells was quite small on pristine ePET (see “AM”), but significantly greater on the bioactive coatings, increasing in the order: LP, LP+CS and LP+CS+EEGF ( $<<0.05)$.

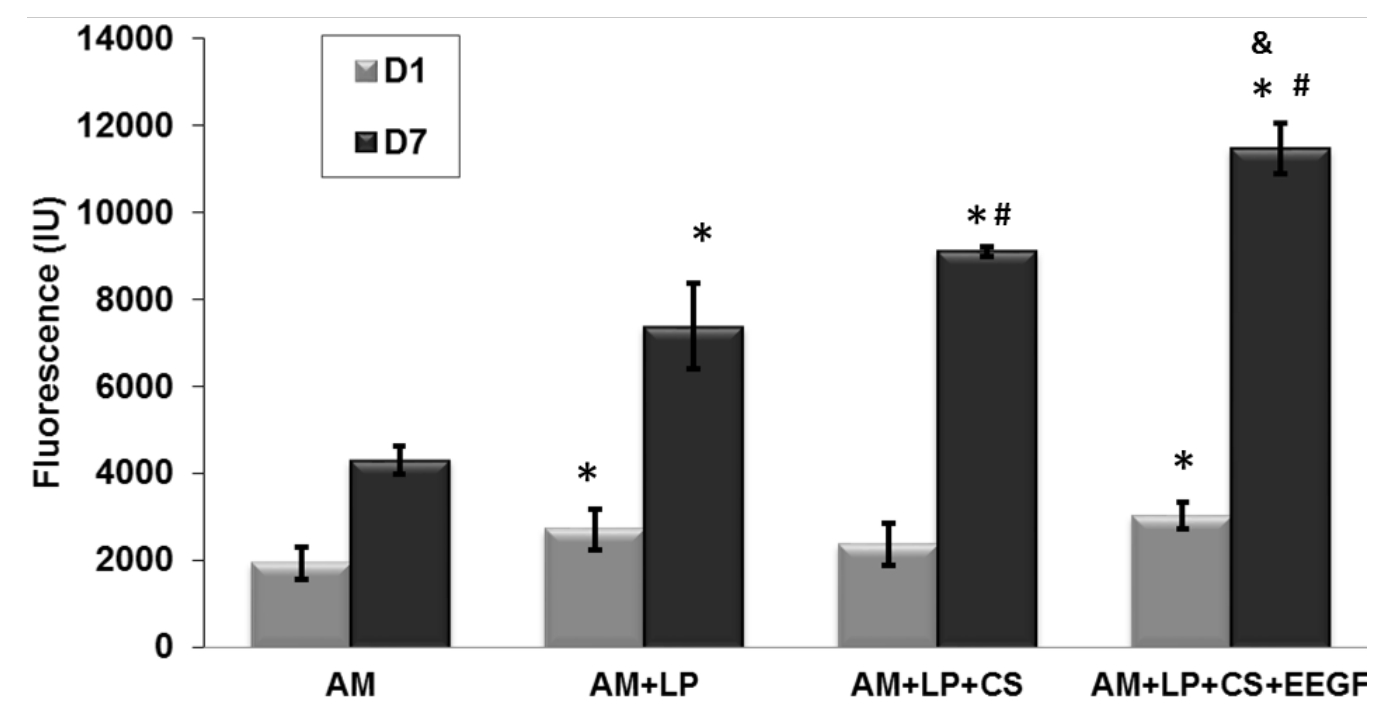

Figure 7 Adhesion after 24 h (D1) and growth after 7 days (D7) of VSMCs on bare and coated substrates [AM: bare aligned mats; ( $\mathrm{n}=8$ for each)].

*, \#, \& $\mathrm{p}<0.05$ with reference to bare, LP, and LP+CS-coated surfaces, respectively. 
Histological examination (Figure 8) confirmed cell infiltration into the bulk of aligned ePET (AM) mats, presumably thanks to their larger pore size, albeit to a limited extent since most cells were encountered on top. Greater penetration might occur by increasing pore size even more or by using dynamic cell seeding.

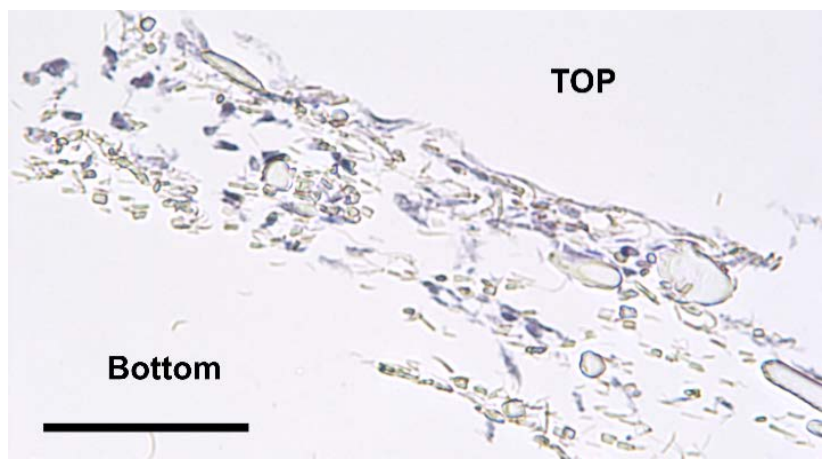

Figure 8 Histological section of an LP-coated AM mat, demonstrating cell infiltration (day 21; H\&E staining, scale bar: $100 \mu \mathrm{m})$.

\subsubsection{VSMC Survival in Serum-Free Medium}

Figure 9 presents VSMC survival after 1, 3 and 5 days in serum-free medium on bare and coated mats, assessed by the AlamarBlue assay. A very strong decrease in cell number is observed after 5 days on bare and LP surfaces (only 5\% survival), indicating that LP did not improve cell survival, even though it increased their initial adhesion. VSMC survival increased on LP+CS (39\% of initial cell count after 5 days; $\mathrm{p}<0.05$ compared to bare and LP), and was still further improved by EGF, $49 \%$ and 55\% of the cells remaining with soluble and immobilized EGF, respectively ( $<<0.05$ compared to LP+CS; without significant difference between immobilized and EGF in solution). This indicated that EGF triggered cell survival mechanisms and that oriented immobilization is at least as efficient as EGF in solution, even though the latter was renewed every 2 days. 


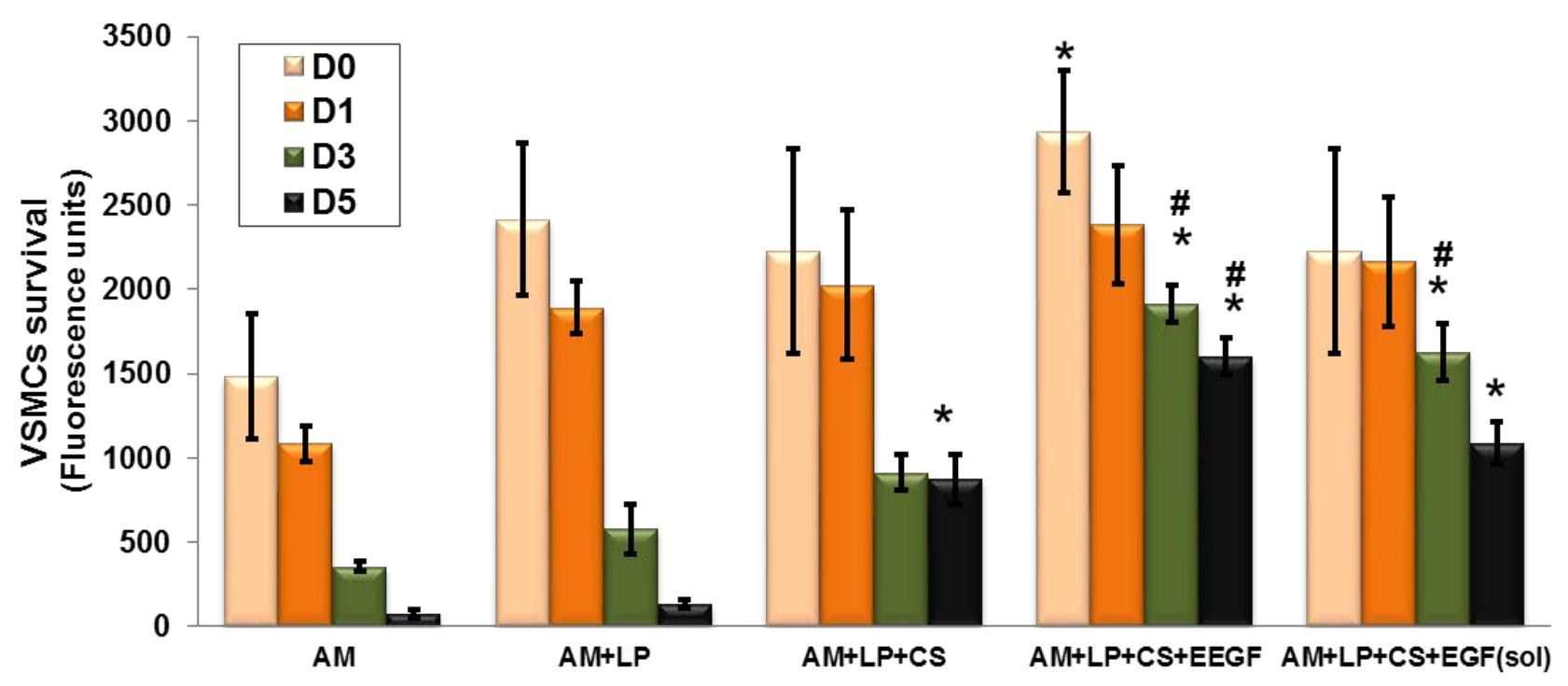

Figure 9 VSMC survival on bare and coated aligned (AM) substrates (LP+CS+EEGF: EGF immobilized on LP+CS-coated mats; AM+LP+CS+EGF(sol); LP+CS-coated mats with soluble

EGF added in medium) (mean + SD; $\mathrm{n}=6$ ). Except LP at D3 and D5, all coatings were significantly better than bare mats at each time point $(\mathrm{p}<0.05$, not indicated on the graph) ${ }^{*} \mathrm{p}<0.05$ compared to LP; ${ }^{\#} \mathrm{p}<0.05$ compared to LP+CS

\subsection{Combining Bioactive Coatings and Electrospun Mats: A Promising Approach for VG}

In this research, we fabricated random and aligned bioactive electrospun PET mats as scaffolds for the luminal and media layers of small-diameter synthetic vascular grafts. Electrospinning enabled us to mimic the ECM morphology, and to control porosity, favouring either monolayer formation (HUVEC) or cell penetration into the bulk (VSMC). We have in addition investigated the effect of fiber alignment, since there is still controversy in the literature regarding the effect of nano-fiber alignment on cell spreading and -proliferation, some studies reporting increased HUVEC growth on aligned mats $[7,10]$ while others show better results with random scaffolds [39]. In the present work, nanofiber alignment was found to have slightly influenced HUVEC alignment and elongation, with minor impact (not significant) on cell retention under flow. The effect of the bioactive coating clearly dominated over the mat topography: Indeed, LP and LP+CS coatings greatly affected cell adhesion, growth and retention on both RL and AL scaffolds. We 
showed that HUVEC adhesion, growth, and retention under flow-induced shear stress were increased by LP coating on ePET mats and smooth PET films [12, 16], but that cell retention was not complete. The present results demonstrated that grafting CS further enhanced HUVEC adhesion, growth and, most important, their resistance to detachment. The fact that CS promotes low platelet adhesion, despite its high propensity for HUVEC adhesion [17] is a clear advantage. Such a non-thrombogenic under-layer could help prevent thrombotic events, should the endothelium lining be incomplete or partially detach under shear-flow. In contrast, other coatings such as simple LP, fibronectin or collagen proposed in the literature are thrombogenic [40]. Thanks to its low thrombogenicity, the potential of our coating to capture in situ endothelial progenitor cells (EPC) to form a complete monolayer on the lumen could also be studied, because it offers a promising alternative to in vitro pre-seeding of cells [40].

The LP+CS coating was also found to be an excellent coating for the medial layer of vascular grafts of tissue constructs. In addition, the oriented immobilization of EGF enabled strong increase in VSMC survival in serum-free media, mimicking the lack of nutrients on the abluminal side of the graft. Immobilization of biomolecules has demonstrated great promise by their resulting durability and stability, which in turn leads to enhanced and continuous cell response [41, 42].

An additional advantage of these coatings is their versatility, since they can be created on any electrospun material thanks to the plasma polymerization process, in contrast to chemical modifications that require optimization as a function of substrate chemistry. In this project, electrospun PET was used for practical and regulatory reasons. However their mechanical properties may not be ideal in regard to compliance. Our previous studies [12, 14] have shown that the Young's moduli of ePET mats, random and aligned, were respectively about $20 \mathrm{MPa}$ and 100 MPa, well above those of natural arteries. Compliance being indirectly related to Young's modulus, we would expect that of the present electrospun mats to be low. We already proposed to resolve this particular issue by chemical means [11] (hydrolysis with concentrated $\mathrm{NaOH}$ ), or by plasma etching [14], if required. But other electrospun materials such as PU or PU/PCL mixture may be used instead [43]. 


\section{Conclusions}

In this project, the combination of electrospinning, plasma polymerization and CS grafting was found to promote the formation of a stable endothelial layer. CS is particularly interesting because it is stable, biocompatible and it prevents platelet adhesion, a key issue to avoid blood clot formation in case of an incomplete EC monolayer or partial cell detachment. Moreover, the versatility of these technologies enables one to adapt them to other polymeric biomaterials, in order to optimize compliance and biodegradation rate, depending on the application. While further work is needed to combine the luminal and medial layers, and to optimize the material for compliance, the present results suggest that the combination of electrospinning and bioactive coatings can provide adequate scaffolds for small-diameter vascular prostheses.

\section{Supporting Information}

Cumultive data for endothelial cell orientation on aligned (AL) mats (Table E1), Typical 2D FFT alignment plot of aligned mats (Figure E1), Histogram of endothelial cell orientation on aligned (AL) mats (Figure E2), Apparent surface densities (absorbance) of oriented tethered EGF, measured by direct ELISA; $\mathrm{p}<0.05$ compared to all other surfaces (n=3) (Figure E3).

\section{Acknowledgments}

This research was supported by grants from the Natural Sciences and Engineering Research Council of Canada (NSERC) and the Canadian Institutes of Health Research (CIHR) (CPG 127764). H.S. gratefully acknowledges the Merit Scholarship from the Fonds de recherche du Québec-Nature et technologies (FQRNT) and an award from the Jane and Frank Warchol Fund of the Society of Vacuum Coaters (SVC) Foundation. The authors also thank C. Cerclé, Dr. J. Lefebvre, Y. Leblanc (École Polytechnique), Micheline Fortin (IRIC-Histology) and the CR CHUM Microscopy Platform for skilled technical support and help during some of the experiments. 


\section{Supporting Information}

\section{Supplementary Table}

Table E1: Cumultive data for endothelial cell orientation on aligned (AL) mats

\begin{tabular}{ccc}
\hline Fiber angle & Frequency & Cumulative \% \\
\hline 0 & 0 & $0.00 \%$ \\
10 & 38 & $37 \%$ \\
20 & 32 & $67 \%$ \\
30 & 15 & $82 \%$ \\
40 & 11 & $92 \%$ \\
50 & 4 & $96 \%$ \\
60 & 1 & $97 \%$ \\
70 & 1 & $98 \%$ \\
80 & 2 & $100 \%$ \\
90 & 0 & $100 \%$ \\
More & 0 & $100 \%$ \\
\hline
\end{tabular}




\section{Supplementary Figures}

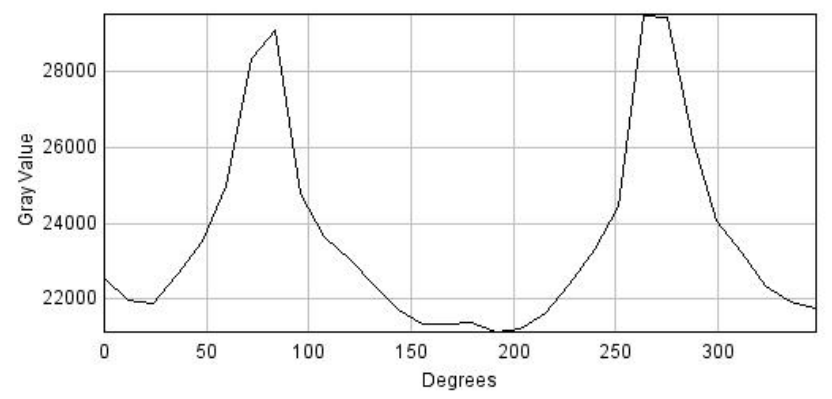

Figure E1: Typical 2D FFT alignment plot of aligned mats.

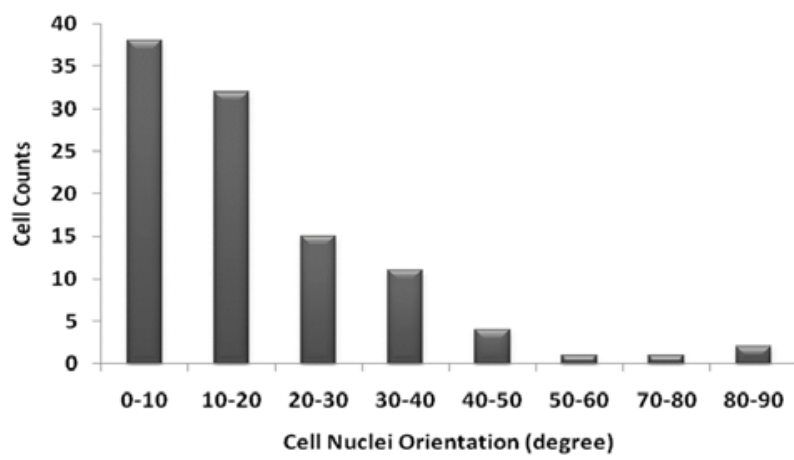

Figure E2: Histogram of endothelial cell orientation on aligned (AL) mats. 


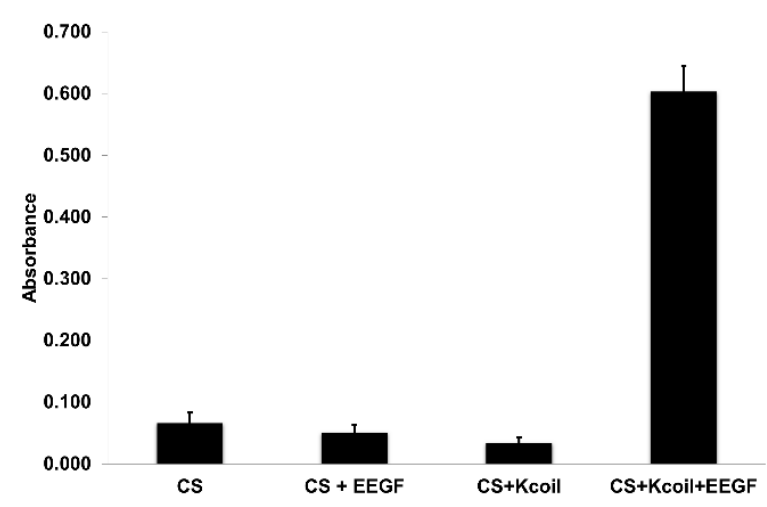

Figure E3: Apparent surface densities (absorbance) of oriented tethered EGF, measured by direct ELISA; $\mathrm{p}<0.05$ compared to all other surfaces $(\mathrm{n}=3)$.

\section{References}

[1] D.G. Seifu, A. Purnama, K. Mequanint, D. Mantovani, Small-diameter vascular tissue engineering, Nature Reviews Cardiology 10(7) (2013) 410-421.

[2] S.L. Dahl, A.P. Kypson, J.H. Lawson, J.L. Blum, J.T. Strader, Y. Li, R.J. Manson, W.E. Tente, L. DiBernardo, M.T. Hensley, Readily available tissue-engineered vascular grafts, Science Translational Medicine 3(68) (2011) 68ra9-68ra9.

[3] N. L'Heureux, N. Dusserre, G. Konig, B. Victor, P. Keire, T.N. Wight, N.A. Chronos, A.E. Kyles, C.R. Gregory, G. Hoyt, Human tissue-engineered blood vessels for adult arterial revascularization, Nature Medicine 12(3) (2006) 361-365.

[4] D.-H. Kim, P.P. Provenzano, C.L. Smith, A. Levchenko, Matrix nanotopography as a regulator of cell function, The Journal of Cell Biology 197(3) (2012) 351-360.

[5] M.W. Tibbitt, K.S. Anseth, Dynamic microenvironments: the fourth dimension, Science Translational Medicine 4(160) (2012) 160ps24-160ps24.

[6] A. Hasan, A. Memic, N. Annabi, M. Hossain, A. Paul, M.R. Dokmeci, F. Dehghani, A. Khademhosseini, Electrospun scaffolds for tissue engineering of vascular grafts, Acta Biomaterialia 10(1) (2014) 11-25.

[7] B.M. Whited, M.N. Rylander, The influence of electrospun scaffold topography on endothelial cell morphology, alignment, and adhesion in response to fluid flow, Biotechnology and Bioengineering 111(1) (2014) 184-195. 
[8] S. Hom, J. O'Hara, W. Yin, D. Rubenstein, Effect of Electrospun Scaffold Fiber Alignment on Endothelial Cell Growth, The FASEB Journal 29(1 Supplement) (2015) 792.2.

[9] H.G. Sundararaghavan, R.L. Saunders, D.A. Hammer, J.A. Burdick, Fiber alignment directs cell motility over chemotactic gradients, Biotechnology and Bioengineering 110(4) (2013) 12491254.

[10] A.K. Gaharwar, M. Nikkhah, S. Sant, A. Khademhosseini, Anisotropic poly (glycerol sebacate)-poly (€-caprolactone) electrospun fibers promote endothelial cell guidance, Biofabrication 7(1) (2014) 015001.

[11] A. Hadjizadeh, A. Ajji, M. Jolicoeur, B. Liberelle, G. De Crescenzo, Effects of electrospun nanostructure versus microstructure on human aortic endothelial cell behavior, Journal of Biomedical Nanotechnology 9(7) (2013) 1195-1209.

[12] H. Savoji, A. Hadjizadeh, M. Maire, A. Ajji, M.R. Wertheimer, S. Lerouge, Electrospun nanofiber scaffolds and plasma polymerization: a promising combination towards complete, stable endothelial lining for vascular grafts, Macromolecular Bioscience 14(8) (2014) 1084-1095.

[13] A. Hadjizadeh, A. Ajji, M.N. Bureau, Nano/micro electro-spun polyethylene terephthalate fibrous mat preparation and characterization, Journal of the Mechanical Behavior of Biomedical Materials 4(3) (2011) 340-351.

[14] H. Savoji, S. Lerouge, A. Ajji, M.R. Wertheimer, Plasma-etching for controlled modification of structural and mechanical properties of electrospun PET scaffolds, Plasma Processes and Polymers 12(4) (2015) 314-327.

[15] J.M. Goddard, J. Hotchkiss, Polymer surface modification for the attachment of bioactive compounds, Progress in Polymer Science 32(7) (2007) 698-725.

[16] A. Gigout, J.C. Ruiz, M.R. Wertheimer, M. Jolicoeur, S. Lerouge, Nitrogen-Rich PlasmaPolymerized Coatings on PET and PTFE Surfaces Improve Endothelial Cell Attachment and Resistance to Shear Flow, Macromolecular Bioscience 11(8) (2011) 1110-1119.

[17] P.K. Thalla, H. Fadlallah, B. Liberelle, P. Lequoy, G. De Crescenzo, Y. Merhi, S. Lerouge, Chondroitin sulfate coatings display low platelet but high endothelial cell adhesive properties favorable for vascular implants, Biomacromolecules 15(7) (2014) 2512-2520.

[18] C. Charbonneau, J.E. Gautrot, M.J. Hébert, X. Zhu, S. Lerouge, Chondroitin-4-Sulfate: A Bioactive Macromolecule to Foster Vascular Healing around Stent-Grafts after Endovascular Aneurysm Repair, Macromolecular Bioscience 7(5) (2007) 746-752.

[19] R.A. Scott, A. Panitch, Glycosaminoglycans in biomedicine, Wiley Interdisciplinary Reviews: Nanomedicine and Nanobiotechnology 5(4) (2013) 388-398.

[20] M.K. O'Connell, S. Murthy, S. Phan, C. Xu, J. Buchanan, R. Spilker, R.L. Dalman, C.K. Zarins, W. Denk, C.A. Taylor, The three-dimensional micro-and nanostructure of the aortic medial lamellar unit measured using 3D confocal and electron microscopy imaging, Matrix Biology 27(3) (2008) 171-181.

[21] P. Lacolley, V. Regnault, A. Nicoletti, Z. Li, J.-B. Michel, The vascular smooth muscle cell in arterial pathology: a cell that can take on multiple roles, Cardiovascular Research 95(2) (2012) 194-204. 
[22] P. Lequoy, B. Liberelle, G. De Crescenzo, S. Lerouge, Additive benefits of chondroitin sulfate and oriented tethered epidermal growth factor for vascular smooth muscle cell survival, Macromolecular Bioscience 14(5) (2014) 720-730.

[23] F. Murschel, B. Liberelle, G. St-Laurent, M. Jolicoeur, Y. Durocher, G. De Crescenzo, Coiled-coil-mediated grafting of bioactive vascular endothelial growth factor, Acta Biomaterialia 9(6) (2013) 6806-6813.

[24] C. Charbonneau, B. Liberelle, M.-J. Hébert, G. De Crescenzo, S. Lerouge, Stimulation of cell growth and resistance to apoptosis in vascular smooth muscle cells on a chondroitin sulfate/epidermal growth factor coating, Biomaterials 32(6) (2011) 1591-1600.

[25] F. Truica-Marasescu, M.R. Wertheimer, Nitrogen-Rich Plasma-Polymer Films for Biomedical Applications, Plasma Processes and Polymers 5(1) (2008) 44-57.

[26] J.C. Ruiz, A. St-Georges-Robillard, C. Thérésy, S. Lerouge, M.R. Wertheimer, Fabrication and Characterisation of Amine-Rich Organic Thin Films: Focus on Stability, Plasma Processes and Polymers 7(9-10) (2010) 737-753.

[27] C. Boucher, J.-C. Ruiz, M. Thibault, M.D. Buschmann, M.R. Wertheimer, M. Jolicoeur, Y. Durocher, G. De Crescenzo, Human corneal epithelial cell response to epidermal growth factor tethered via coiled-coil interactions, Biomaterials 31(27) (2010) 7021-7031.

[28] B. Liberelle, C. Boucher, J. Chen, M. Jolicoeur, Y. Durocher, G. De Crescenzo, Impact of epidermal growth factor tethering strategy on cellular response, Bioconjugate Chemistry 21(12) (2010) 2257-2266.

[29] S. De Valence, J.-C. Tille, J.-P. Giliberto, W. Mrowczynski, R. Gurny, B. Walpoth, M. Moeller, Advantages of bilayered vascular grafts for surgical applicability and tissue regeneration, Acta Biomaterialia 8(11) (2012) 3914-3920.

[30] P. Lequoy, F. Murschel, B. Liberelle, S. Lerouge, G. De Crescenzo, Controlled coimmobilization of EGF and VEGF to optimize vascular cell survival, Acta Biomaterialia 29 (2016) 239-247.

[31] T. Fujie, X. Shi, S. Ostrovidov, X. Liang, K. Nakajima, Y. Chen, H. Wu, A. Khademhosseini, Spatial coordination of cell orientation directed by nanoribbon sheets, Biomaterials 53 (2015) 8694.

[32] J.L. Charest, A.J. García, W.P. King, Myoblast alignment and differentiation on cell culture substrates with microscale topography and model chemistries, Biomaterials 28(13) (2007) 22022210.

[33] M. Nikkhah, N. Eshak, P. Zorlutuna, N. Annabi, M. Castello, K. Kim, A. Dolatshahi-Pirouz, F. Edalat, H. Bae, Y. Yang, Directed endothelial cell morphogenesis in micropatterned gelatin methacrylate hydrogels, Biomaterials 33(35) (2012) 9009-9018.

[34] J.C. Kohn, D.W. Zhou, F. Bordeleau, A.L. Zhou, B.N. Mason, M.J. Mitchell, M.R. King, C.A. Reinhart-King, Cooperative effects of matrix stiffness and fluid shear stress on endothelial cell behavior, Biophysical Journal 108(3) (2015) 471-478.

[35] S. Babaei, P.-L. Girard-Lauriault, Tuning the Surface Properties of Oxygen-Rich and Nitrogen-Rich Plasma Polymers: Functional Groups and Surface Charge, Plasma Chemistry and Plasma Processing 36(2) (2016) 651-666. 
[36] Z. Ma, M. Kotaki, T. Yong, W. He, S. Ramakrishna, Surface engineering of electrospun polyethylene terephthalate (PET) nanofibers towards development of a new material for blood vessel engineering, Biomaterials 26(15) (2005) 2527-2536.

[37] D. Gugutkov, J. Gustavsson, M.P. Ginebra, G. Altankov, Fibrinogen nanofibers for guiding endothelial cell behavior, Biomaterials Science 1(10) (2013) 1065-1073.

[38] B. Bondar, S. Fuchs, A. Motta, C. Migliaresi, C.J. Kirkpatrick, Functionality of endothelial cells on silk fibroin nets: comparative study of micro-and nanometric fibre size, Biomaterials 29(5) (2008) 561-572.

[39] D.E. Heath, J.J. Lannutti, S.L. Cooper, Electrospun scaffold topography affects endothelial cell proliferation, metabolic activity, and morphology, Journal of Biomedical Materials Research Part A 94(4) (2010) 1195-1204.

[40] S. Li, J.J.D. Henry, Nonthrombogenic Approaches to Cardiovascular Bioengineering, Annual Review of Biomedical Engineering 13(1) (2011) 451-475.

[41] B. Joddar, Y. Ito, Biological modifications of materials surfaces with proteins for regenerative medicine, Journal of Materials Chemistry 21(36) (2011) 13737-13755.

[42] P. Lequoy, H. Savoji, B. Saoudi, A. Bertrand-Grenier, M.R. Wertheimer, G. De Crescenzo, G. Soulez, S. Lerouge, In Vitro and Pilot In Vivo Evaluation of a Bioactive Coating for Stent Grafts Based on Chondroitin Sulfate and Epidermal Growth Factor, Journal of Vascular and Interventional Radiology 27(5) (2016) 753-760. e3.

[43] F. Guo, N. Wang, L. Wang, L. Hou, L. Ma, J. Liu, Y. Chen, B. Fan, Y. Zhao, An electrospun strong PCL/PU composite vascular graft with mechanical anisotropy and cyclic stability, Journal of Materials Chemistry A 3(9) (2015) 4782-4787. 